\title{
Advancing progress for patients with cancer through small business innovation research
}

\author{
Norman E. Sharpless
}

National Cancer Institute, NIH, Bethesda, Maryland, USA.

A $s$ the federal government's principal agency for cancer research, the National Cancer Institute (NCI) leads many activities related to basic research, clinical trials, training, and information dissemination.

Perhaps less well known are NCI's Small Business Innovation Research (SBIR) and Small Business Technology Transfer (STTR) programs, which have been in existence for several decades and specifically set up as America's seed fund $(1,2)$. SBIR/STTR programs are designed to provide startup funds to small businesses to help them develop and commercialize innovative technologies - devices, diagnostics, algorithms, and therapeutics - to improve cancer prevention, detection, and care. As an academic scientist, I helped a few small companies obtain SBIR funding to commercialize science from my lab and found these grants highly effective for fostering translational research

By statute, all federal agencies that provide at least $\$ 100$ million in annual funding for research and development are required to set aside a portion of their extramural research budget (3.65\% in 2020) for SBIR/STTR activities. In fiscal year (FY) 2020, the set-aside for the NCI's extramural budget is approximately $\$ 179$ million, including Cancer Moonshot funding, for the development of the next generation of technologies. This is one of the largest and best sources of financing for early-stage development of cancer biotechnology in the United States.

\section{SBIR/STTR fills a critical technology development gap}

NCI's support of small business differs from that of the private sector. Private sector investors tend to invest in businesses with an established track record. NCI, on the other hand, is willing to take on more risk, funding innovative projects at an early, proof-of-concept stage. Additionally, private capital tends to flow preferentially toward technologies (e.g., novel therapeutics) with the highest potential for return on investment, whereas NCI can invest in technologies (e.g., devices and diagnostics) with a less straightforward path to commercialization, but where patient needs are just as significant. These funds are essential for helping projects advance to a stage at which private companies would be more interested in investing in further commercialization of these new technologies.

NCI funding is an extremely beneficial arrangement for startups because it is "nondilutive," meaning the small business is not required to give up equity positions in its company. The intellectual property remains fully owned by the business, and there are no repayment conditions to the government.

SBIR/STTR offers funding opportunities for three phases of product development (Figure 1). Phase I is the initial feasibility/proof-of-concept phase, with a duration generally up to one year, and awards up to a maximum of $\$ 400,000$. Should the technology prove successful SBIR/STTR grantees may apply for Phase II funding, a fuller research-and-development phase with awards of up to $\$ 2$ million.

The next support phase, the Phase IIB Bridge award, is designed to help successful Phase II SBIR/STTR grantees accelerate the commercialization of their projects. The award addresses the critical period when many companies fail, commonly referred to as the "Valley of Death." It was specifically instituted to deal with startups' need for resources during this period, when they are seeking to advance

Conflict of interest: The author has declared that no conflict of interest exists.

Copyright: @ 2020, American Society for Clinical Investigation.

Reference information: / Clin Invest. 2020;130(7):3339-3341. https://doi.org/10.1172/JCI138643.

their technology to a key inflection pointtypically into human clinical trials.

The Phase IIB Bridge awards provide a maximum of $\$ 4$ million for up to three years. Because NCI has already helped decrease the risk of failure of these technologies by funding them at their earliest stages, there is a greater likelihood that investors and/or strategic partners would be willing to invest alongside $\mathrm{NCI}$ in these more mature projects. Applicants for the Phase IIB Bridge awards are expected to raise matching funds from private sector investors, and the strength of their fundraising plans is a key component of peer review. This Bridge funding has been very successful; of 35 projects that were funded since the award was created ten years ago, 14 commercial products have been launched. Thus far, companies have been able to raise, on average, $\$ 4$ in third-party funding for every $\$ 1$ in NCI funding.

\section{A seamless continuum of support for small businesses}

Funds are a crucial ingredient that can influence a startup's chances of success, but on their own, they are insufficient. Accordingly, SBIR/STTR has put in place many innovative entrepreneurship training initiatives for grantees to increase the likelihood of their technologies reaching the commercialization stage.

The goal is to offer a seamless continuum of tools and resources from early mentoring assistance with applications for SBIR/STTR funds, to entrepreneurship training on how to build a business model around their technology, to access to investors and strategic partners not otherwise available to them to help commercialize a promising biomedical technology.

Since the inception of SBIR/STTR, the $\mathrm{NCI}$ has continued to assess the programs, looking for gaps where additional resources and/or funding are needed to foster a startup's success in developing a technology to the point of commercialization. 


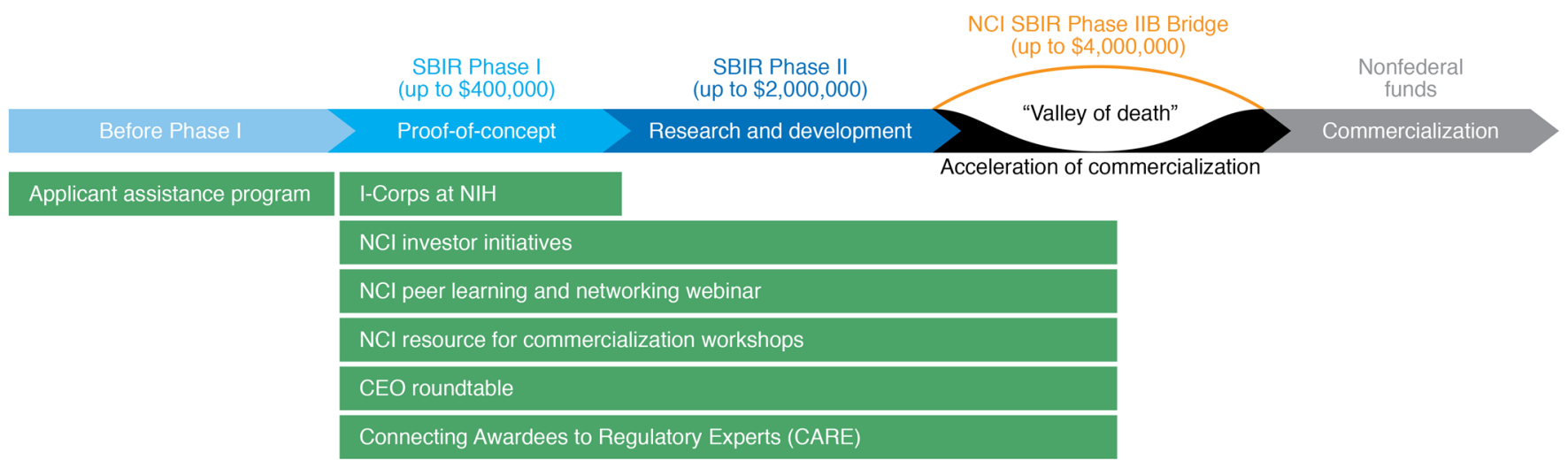

Figure 1. Commercialization resources available at NCI SBIR Development Center. SBIR/STTR offers funding opportunities for 3 phases of technology development. Phase I is the initial proof-of-concept phase. Phase II is a fuller research-and-development stage of successful Phase I projects. The Phase IIB Bridge facilitates acceleration of project commercialization, a critical period commonly referred to as the "Valley of Death," during which many companies fail. The Bridge was specifically instituted to deal with startups' need for resources to advance their technology to a key inflection point - typically into human clinical trials. As early investment from the $\mathrm{NCl}$ has decreased the risk of failure for projects entering Phase IIB, projects have a high success rate for commercialization. Of note, the major distinguishing feature of the STTR program from the SBIR program is that projects must involve a cooperative research-and-development arrangement between small businesses and research institutions. Additionally, the project director/principal investigator in the STTR program is not required to have his/her main employment from the small business for the duration of the grant. For more information on these programs and resources, see https://sbir.cancer.gov/programseducation.

\section{Impact of SBIR/STTR programs on the broader community}

To better understand the impact of NCI's SBIR/STTR programs on the broader community and how the program might be strengthened, NCI commissioned an economic impact assessment of the program's overall contribution to cancer research and the national economy leading up to 2018 (3). The study focused on Phase II investments from 1998 through 2010. A few key findings include the following: (a) NCI invested $\$ 787$ million of Phase II SBIR/STTR funds in 444 companies for 690 separate projects, resulting in 247 technologies that were commercially available as of 2018, including 71 FDA-approved products; (b) the SBIR/ STTR investments translated into an estimated $\$ 26.1$ billion in total economic output nationwide, including product sales, tax revenue, and job creation, which is a 33:1 return on investment; and (c) approximately $65 \%$ of the surveyed technologies supported by the SBIR/STTR programs were developing a treatment option for a group of patients who previously lacked treatment options.

These results demonstrate that SBIR/ STTR efforts to nurture commercialization of cutting-edge cancer science are not only economically significant, but also provide a much-needed means of getting novel technologies from the research bench into broad clinical use in a way that will directly help patients with cancer.

\section{A new technology to detect early-stage lung cancer}

Many success stories are evident among the small businesses that SBIR/STTR has supported over the years. One example is Percepta, a practice-changing tool for lung cancer screening and detection. In this case, NCI academic and small business grants both played important roles in translating biological research into commercial technology that is now benefiting people in the clinical setting.

Avrum Spira, physician, researcher, entrepreneur, and the Global Head of the Lung Cancer Initiative at Johnson \& Johnson, began working on this new lung cancer-screening and-detection technology more than a decade ago. At that time, while supported by an NCI R01 grant, he was investigating genomic changes in the airways of patients with lung cancer.

Spira and his team identified a genetic signature that could reveal the presence of early-stage lung cancer, a finding that led him to the idea of collecting cells in the airway and testing them for the signature. He then developed a minimally invasive way to detect the changes indicating early-stage lung cancer in patients by equipping a bronchoscope with a brush that could collect airway cells, which would then be analyzed for these molecular changes.

To test this new bronchial genomic classifier, Spira would need to conduct the necessary clinical trials. SBIR funding provided the critical capital for clinical trials that would test this early-stage technology. The trials validated the usefulness of the tool in determining the likelihood that lung nodules were either benign or malignant. A year later, Spira's company was acquired by Veracyte, a genomic diagnostics company, which commercially launched the new device that is now used in clinics and covered by Medicare (4).

\section{Continuous improvement}

With the goal of continuous improvement to its programs, I recently convened a working group of National Cancer Advisory Board (NCAB) members and leaders from academia, the pharmaceutical industry, and the venture capital community to evaluate the SBIR/STTR programs (5).

The NCAB working group gave positive reviews of the SBIR/STTR programs and provided recommendations on ways to enhance the programs moving forward in areas of improving process and enhancing diversity among applicants - recommendations that NCI has begun implementing.

The working group's top recommendation was to establish a high-risk, high-reward concept award for fostering 
"disruptive innovation." The idea behind the award would be to raise the bar on innovation and support small businesses in the development of significantly transformative technologies that help cancer patients, providers, and caregivers. This award would provide funds for startups to undertake key experiments that would provide the foundation for a follow-up high-impact Phase I grant application.

Other recommendations from the working group included broadening geographic, sex, and racial/ethnic diversity of participants and peer reviewers; increasing award size; enhancing application assistance and entrepreneurship training for grantees; improving metrics for continuous evaluation; and expanding outreach and awareness of the programs.

\section{Fostering diversity}

Too few SBIR/STTR awards go to companies with female or underrepresented minority founders. To foster greater participation in technological innovation by socially and economically disadvantaged small businesses and by women-owned or -led small businesses, SBIR/STTR recently initiated the Applicant Assistance Program (AAP) (6). The AAP provides coaching and mentoring to help small businesses prepare and submit applications for SBIR/ STTR funds. NCI is encouraging companies with women and individuals from underrepresented populations in leadership positions to apply to the AAP. Approximately $64 \%$ of the AAP participants were from women-owned businesses.

The AAP appears to be a useful program in encouraging applications to the SBIR/STTR program. What we have seen so far is that $85 \%$ of the small businesses who participated in the AAP submitted an application to SBIR/STTR at the end of the program. In contrast, just $15 \%$ of the small businesses who were not selected to participate in the AAP submitted an
SBIR/STTR application by the next receipt date. In addition, when AAP participants were compared with first-time applicants to SBIR/STTR, AAP participants had a higher success rate in securing funds, indicating that the AAP may help close the funding gap that currently exists between underrepresented and non-underrepresented small businesses.

Another endeavor that is making a big difference in supporting women as entrepreneurs is I-Corps at NIH (7). In FY 2017, $61 \%$ of I-Corps awardees included women on the team.

Designed as an entrepreneurial training program, I-Corps offers training, mentoring, and networking opportunities to help small businesses commercialize promising biomedical technology. During the 8-week, hands-on program, awardees are required to conduct a minimum of 100 interviews with potential customers, strategic partners, and other thirdparty stakeholders to help them maximize their understanding of the market for their invention, identify regulatory and commercial pathways to advance their technology, and focus their business plan. Graduates of the I-Corps program have consistently remarked that the I-Corps program empowered them with invaluable entrepreneurial knowledge that, as bench scientists, they would not have otherwise gained through their work.

\section{Moving science into clinics for the benefit of patients}

Since inception, NCI's SBIR/STTR programs have supported thousands of small US businesses, some of which have been highly successful and are now large US businesses (or have been acquired by large businesses). By providing nondilutive funding and other resources to small biotech companies during the early stages of development, the programs have helped to transform scientists into entre- preneurs who know what technologies are most needed to help patients, providers, and caregivers and who understand how to navigate the commercialization steps necessary to bring their technologies to market. SBIR/STTR's support of small business innovation plays a critical role in helping NCI move a step closer to fulfilling its mission to advance scientific knowledge and help all people live longer, healthier lives.

\section{Acknowledgments}

I am grateful to Michael Weingarten for advice on the manuscript.

Address correspondence to: Norman E. Sharpless, National Cancer Institute, National Institutes of Health, 31 Center Drive, MSC 2590, Building 31, Room 11A48, Bethesda, Maryland 20892-2590, USA. Email: Norman.Sharpless@nih.gov.

1. NIH. SBIR Development Center. National Cancer Institute. https://sbir.cancer.gov. Accessed May 27, 2020.

2. SBIR/STTR. America's Seed Fund. https://www. sbir.gov. Accessed May 27, 2020.

3. NIH. SBIR Development Center. SBIR Impact Study. National Cancer Institute. https://sbir. cancer.gov/impact. Updated May 10, 2019. Accessed May 27, 2020.

4. NIH. Brushing the Airway to Detect Lung Cancer Earlier. National Cancer Institute. https://www. cancer.gov/research/areas/screening/avrumspira-early-detection-lung-cancer. Updated September 21, 2017. Accessed May 27, 2020.

5. National Cancer Advisory Board Ad Hoc Working Group on SBIR/STTR. National Cancer Advisory Board Ad Hoc Working Group Report of the National Cancer Institute Small Business Innovation Research Program. National Cancer Institute. https://deainfo.nci.nih.gov/advisory/ ncab/workgroup/SBIRSTTR/FinalReport 05Feb2019.pdf. Accessed May 27, 2020.

6. NIH. Applicant Assistance Program. National Cancer Institute. https://sbir.cancer.gov/ programseducation/aap. Accessed May 27, 2020.

7. NIH. SBIR Development Center. I-Corps at NIH. National Cancer Institute. https://sbir. cancer.gov/programseducation/icorps. Accessed May 27, 2020. 